\title{
Subject Name
}

National Cancer Institute

\section{Source}

National Cancer Institute. Subject Name. NCI Thesaurus. Code C69257.

The name of the individual of interest. 